FOLIA

Amazónica

Revista del Instituto de Investigaciones

de la Amazonía Peruana

\title{
VIABILIDAD ECONÓMICA Y FINANCIERA DEL CULTIVO DE ARROZ (Oryza sativa L.) Y YUCA (Manihot esculenta Crantz), EN UN SISTEMA DE PRODUCCIÓN DE PIJUAYO (Bactris gasipaes Kunth) EN LORETO, PERÚ
}

\author{
Elías CUMARI LAULATE ${ }^{1}$, Juan Imerio URRELO CORREA ${ }^{1}$, \\ Rodrigo GONZÁLES VEGA²
}

1 Universidad Nacional de la Amazonía Peruana, Facultad de Agronomía, lquitos, Perú.

2 Instituto Nacional de Investigación Agraria. Estación Experimental Agraria San Roque, San Juan Bautista, Iquitos, Perú. Correo electrónico: rgonzalesv@inia.gob.pe

\section{RESUMEN}

El objetivo del estudio fue determinar la viabilidad económica y financiera de un sistema productivo de pijuayo (Bactris gasipaes Kunth) para fruto, asociado de manera secuencial con arroz (Oryza sativa L.) y yuca (Manihot esculenta Crantz). La investigación se desarrolló en tres parcelas experimentales ubicadas a lo largo de la carretera Iquitos-Nauta, Loreto, Perú: Campo experimental El Dorado km 25,5; Parcela experimental El Paujil km 35,5 y Parcela experimental Bolloquito $\mathrm{km} 75,5$. Fue empleado el método analítico relacional para evaluar los resultados económicos y financieros de los componentes arroz y yuca en un sistema de producción de pijuayo para fruto (04 genotipos, más un testigo, genotipo local), distribuidos en tres bloques al azar. Encontramos que los componentes arroz y yuca se muestran económicamente lucrativos, donde la parcela de El Dorado resultó tener mayor rentabilidad. En el sistema productivo de pijuayo para fruto, el arroz y la yuca hacen posible los ingresos económicos suficientes durante los primeros años. Por lo tanto, el sistema agrario productivo de pijuayo, asociados con arroz y yuca es viable económica y financieramente.

PALABRAS CLAVE: rentabilidad, sistemas agroforestales, productividad, rendimiento. 


\title{
ECONOMIC VIABILITY OF RICE (Oryza sativa L.) AND CASSAVA (Manihot esculenta Crantz), IN A PRODUCTION SYSTEM OF PIJUAYO (Bactris gasipaes Kunth) IN LORETO, PERU
}

\begin{abstract}
The objective of the study was to determine the economic viability of a productive system of pijuayo (Bactris gasipaes Kunth) for fruit, associated with rice (Oryza sativa L.) and cassava (Manihot esculenta Crantz). The research was carried out in three experimental plots located along Iquitos-Nauta highway, Loreto, Peru: 1) Experimental Field El Dorado km 25.5, 2) El Paujil Experimental Plot km 35.5, and 3) Experimental Plot Bolloquito km 75.5. A relational analysis was employed to assess the economic viability. Rice and cassava components of the Pijuayo fruit production system were distributed in three random blocks (04 genotypes, plus one control, local genotype). We found that rice and cassava were economically profitable. The plot of El Dorado having the highest profitability. Thus, in the Pijuayo fruit productive system, rice and cassava may provide sufficient economic income in the first years. Therefore, the productive agrarian system of pijuayo associated with rice and cassava is economically and financially viable.
\end{abstract}

KEYWORDS: Profitability, agroforestry systems, productivity, yield. 


\section{INTRODUCCIÓN}

El Perú se ubica en el décimo puesto en el ranking mundial de áreas con mayor densidad forestal, muchas personas dependen directamente de estos bosques para su subsistencia, causando su deforestación, principalmente por la agricultura, ganadería, minería comercial y construcción de vías de transporte; estos aspectos favorecieron para incluir a la Amazonía en la lista de las principales regiones en peligro de deforestación (Smith \& Schwartz, 2015; SERFOR, 2015). En la región Loreto, el incremento de la deforestación para el periodo 2000-2014 alcanzó un total 294 793,18 ha; siendo la provincia de Alto Amazonas la más deforestada con 78 878,83 (Urquiza \& Burga, 2016). Ante esta acelerada destrucción de los bosques tropicales amazónicos, es necesaria la búsqueda de nuevas estrategias y sistemas productivos amazónicos, a fin de atenuar el creciente deterioro de los recursos naturales. Los sistemas de producción en la Amazonía se desarrollan básicamente en la ribera de los ríos, desde tiempos inmemoriales, por los pobladores nativos a escala familiar con fines de subsistencia y escaso nivel de rentabilidad, siendo el arroz (Oryza sativa L.) el más rentable (Chibnik, 1994; List et al., 2019). Sin embargo, la agricultura ribereña está expuesta a una serie de influencias físicas y económicas que afectan sus niveles de producción (Labarta et al., 2007). Los sistemas agroforestales surgen como una alternativa en la Amazonía, durante el periodo 2011-2015, el PEDICP, incentivó la instalación de 2000 hectáreas de sistemas agroforestales, donde destacan los cultivos anuales de yuca, plátano y arroz, y teniendo al cacao y plantones forestales como principales componentes del sistema (MINAGRI, 2016). El arroz, es uno de los alimentos básicos del poblador peruano, cubre el $19 \%$ del total diario de calorías que se requiere, y la producción nacional abastece la totalidad de la demanda interna (Palacios, 2010). La yuca (Manihot esculenta Crantz), de alta producción de raíces con reservas, es la mejor fuente de carbohidratos y proteínas en los sistemas de producción (Martínez et al., 2006), constituye uno de los alimentos fundamentales, especialmente en aquellas zonas con déficit alimentario (INIA, 2014). En los sistemas secuenciales, los cultivos anuales y arbóreos se relevan en el mismo espacio (Mao et al., 2000; Rivas, 2005), existiendo una relación cronológica, donde la secuencia en el tiempo mantiene la competencia al mínimo (Musalem, 2001). La utilidad de la evaluación financiera radica en determinar si el proyecto es rentable en términos de costos y beneficios para los productores (Álvarez \& Peña, 2003). Además, genera información necesaria para comparar el proyecto con otras oportunidades de inversión (Girón, 2012). Por lo tanto, el presente estudio tuvo como propósito evaluar la viabilidad económica-financiera del cultivo de arroz y yuca, en un sistema de producción con pijuayo (Bactris gasipaes Kunth) a lo largo de la carretera Iquitosnauta, en Loreto, Perú.

\section{MATERIAL Y MÉTODOS}

\section{ÁREA DE ESTUDIO}

La investigación se desarrolló en tres parcelas experimentales ubicadas a lo largo de la carretera Iquitos-Nauta, Loreto, Perú. El Campo Experimental El Dorado km 25,5 INIA-San Roque, localizado en las coordenadas geográficas $03^{\circ} 57^{\prime} 26^{\prime \prime}$ S, 73 $24^{\prime} 23^{\prime \prime} \mathrm{W}$, a una altitud de 139 msnm. Parcela experimental El Paujil km 35,5, localizado en las coordenadas $04^{\circ} 00^{\prime} 42^{\prime \prime} \mathrm{S}$; $73^{\circ} 28^{\prime} 03^{\prime \prime}$ W, a una altitud de $129 \mathrm{msnm}$. Parcela experimental Bolloquito km 75,5 localizada en las coordenadas $04^{\circ} 22^{\prime} 21^{\prime \prime} \mathrm{S}$; $73^{\circ} 33^{\prime} 44^{\prime \prime} \mathrm{W}$, a una 
altitud de 126 msnm. Según Holdridge (1987), las localidades donde se desarrolló la investigación, están clasificadas como bosque húmedo tropical, caracterizado por sus altas temperaturas, superiores a $26{ }^{\circ} \mathrm{C}$ y fuertes precipitaciones que oscilan entre $2000 \mathrm{~mm}$ y $4000 \mathrm{~mm}$ por año.

Se utilizó material de propagación de la variedad "La Esperanza" de Oryza sativa L. provenientes de la Estación Experimental Agraria "El Porvenir", resistente a plagas y enfermedades, y esquejes de la variedad "Señorita" de yuca Manihot esculenta Crantz, resistente a plagas y enfermedades, provenientes de parcelas de productores de la zona.

\section{EVALUACIONES}

\section{Valor Actual Neto (VAN)}

Es el valor actual de los beneficios netos que genera el proyecto. Calculado por:

$$
\mathrm{VAN}=\sum_{t=1}^{n} \frac{V t}{(1+k)^{t}}-I_{0}
$$

Dónde: $\mathrm{I}_{0}=$ inversión en el periodo cero, $\mathrm{V}_{\mathrm{t}}=$ beneficios del periodo, $\mathrm{K}=$ tasa de interés o costo de oportunidad del capital.

\section{Tasa Interna de Retorno (TIR)}

La Tasa Interna de Retorno (TIR) es el tipo de descuento que hace igual a cero el VAN:

$$
\mathrm{VAN}=\sum_{t=1}^{n} \frac{F t}{(1+T I R)^{t}}-I=0
$$

Dónde: $\mathrm{Ft}=$ es el flujo de caja en el periodo $\mathrm{t}$, $\mathrm{N}=$ es el número de periodos, I= es el valor de la inversión inicial.

\section{Relación Beneficio Costo (RBC)}

Es el coeficiente de evaluación que resulta de dividir los ingresos netos actualizados entre los egresos netos actualizados a la tasa de interés que representa el costo de oportunidad de capital.
La fórmula que nos permite calcular la RBC es:

$$
\mathrm{RBC}=\frac{V P B}{V P C}
$$

Donde: $\mathrm{RBC}=$ Relación beneficio-costo, $\mathrm{VPB}=$ Valor presente de los beneficios brutos, $\mathrm{BPC}=$ Valor presente de los costos brutos.

\section{ANÁLISIS DE DATOS}

Para el análisis de la viabilidad técnica y financiera de los componentes arroz y yuca del sistema productivo del pijuayo en las tres localidades, se elaboró el flujo de caja de ingresos y egresos. Para los indicadores de rentabilidad financiera se utilizaron las ecuaciones para cálculos del VAN, TIR y la relación B/C, mencionadas anteriormente.

\section{RESULTADOS Y DISCUSIÓN}

INDICADORES DE LA VIABILIDAD ECONÓMICA-FINANCIERA

Para los periodos del cultivo del arroz y cultivo de yuca en la localidad El Dorado, se reporta un flujo de caja positivo de S/ 177,00 para el primero y un flujo de caja también positivo de S/ 9688,51 para el segundo. El VAN muestra una rentabilidad positiva de ganancia en este sistema de S/ 12 559,52, la TIR indica que se puede pagar una tasa de interés de hasta $40,98 \%$, muy superior a la tasa de interés del sistema financiero agrario que es del $15 \%$, el ratio $\mathrm{B} / \mathrm{C}$ resultó positivo, ya que por cada sol $(S / 1,0)$ que se invierte, se recuperan $S / 1,50$ (Tabla 1 ).

Con respecto al flujo de caja para los periodos del cultivo del arroz y del cultivo de yuca en la localidad El Paujil, se reporta un flujo de caja negativo de $S / 319,05$ para el primero y un flujo de caja positivo de S/ 10 402,85 para el segundo. El VAN indica rentabilidad positiva de S/ 12 463,59, la TIR indica que se puede pagar una tasa de interés de 41,09\%, superior a la tasa de interés 
Tabla 1. Flujo de caja económico de los componentes arroz (Oryza sativa L.) y yuca (Manihot esculenta Crantz) del sistema de producción en la localidad de El Dorado, Carretera lquitos-Nauta, Loreto, Perú.

\section{Análisis económicos del cultivo del arroz y yuca en El Dorado}

\begin{tabular}{|c|c|c|c|}
\hline \multicolumn{4}{|c|}{ Cálculo del VAN, RB/C y del TIR con una tasa de descuento del 15\% } \\
\hline FLUJOS/PERIODO & PERIODO 0 & PERIODO 1 & PERIODO 2 \\
\hline Ingresos & 0.00 & 3663,00 & 12397,52 \\
\hline Egresos & 5000,00 & 3486,00 & 2709,00 \\
\hline Flujo de fondos & $-5000,00$ & 177,00 & 9688,51 \\
\hline \multicolumn{4}{|c|}{ Los indicadores económicos que arroja los cultivos en El Dorado } \\
\hline \multicolumn{2}{|c|}{ Tasa de descuento } & \multicolumn{2}{|c|}{$15 \%$} \\
\hline Valor Neto Actual & S/ 12559,52 & \multicolumn{2}{|c|}{ Estimaciones de la viabilidad } \\
\hline Tasa Interna de Retorno & $40,98 \%$ & VPB & S/ 7479,82 \\
\hline Relación Beneficio/Costo & $S / 1,50$ & VPC & S/ 5000,00 \\
\hline \multicolumn{4}{|c|}{ Los indicadores económicos que arrojan los cultivos en El Dorado son: } \\
\hline Valor Neto Actual (VAN) & & Viable & \\
\hline Tasa Interna de Retorno (TIR) & & Viable & \\
\hline Relación Benficio Costo (BC) & & Viable & \\
\hline
\end{tabular}

Tabla 2. Flujo económico de caja de los componentes arroz (Oryza sativa L.) y yuca (Manihot esculenta Crantz) del sistema de producción en la localidad de El Paujil, Carretera lquitos-Nauta, Loreto, Perú.

\begin{tabular}{|c|c|c|c|}
\hline \multicolumn{4}{|c|}{ Análisis económicos del cultivo del arroz y yuca en El Paujil } \\
\hline \multicolumn{4}{|c|}{ Cálculo del VAN, RB/C y del TIR con una tasa de descuento del 15\% } \\
\hline FLUJOS/PERIODO & PERIODO 0 & PERIODO 1 & PERIODO 2 \\
\hline Ingresos & 0.00 & 3000,00 & 13033,52 \\
\hline Egresos & 5000,00 & 3319,00 & 2630,25 \\
\hline Flujo de fondos & $-5000,00$ & $-319,00$ & 10402,85 \\
\hline \multicolumn{4}{|c|}{ Los indicadores económicos que arroja los cultivos en El Paujil } \\
\hline \multicolumn{2}{|c|}{ Tasa de descuento } & \multicolumn{2}{|c|}{$15 \%$} \\
\hline Valor Neto Actual & S/ 12463,59 & \multicolumn{2}{|c|}{ Estimaciones de la viabilidad } \\
\hline Tasa Interna de Retorno & $41,09 \%$ & VPB & $S / 7588,61$ \\
\hline Relación Beneficio/Costo & $\mathrm{S} / 1,52$ & VPC & $\mathrm{S} / 5000,00$ \\
\hline \multicolumn{4}{|c|}{ Los indicadores económicos que arrojan los cultivos en El Paujil son: } \\
\hline Valor Neto Actual (VAN) & & Viable & \\
\hline Tasa Interna de Retorno (TIR) & & Viable & \\
\hline Relación Benficio Costo (BC) & & Viable & \\
\hline
\end{tabular}


Tabla 3. Flujo económico de caja de los componentes arroz (Oryza sativa L.) y yuca (Manihot esculenta Crantz) del sistema de producción en la localidad de El Bolloquito, Carretera lquitos-Nauta, Loreto, Perú.

\begin{tabular}{|c|c|c|c|}
\hline \multicolumn{4}{|c|}{ Análisis económicos del cultivo del arroz y yuca en El Bolloquito } \\
\hline \multicolumn{4}{|c|}{ Cálculo del VAN, RB/C y del TIR con una tasa de descuento del 15\% } \\
\hline FLUJOS/PERIODO & PERIODO 0 & PERIODO 1 & PERIODO 2 \\
\hline Ingresos & 0.00 & 2460,00 & 10804,05 \\
\hline Egresos & 5000,00 & 2672,25 & 2425,50 \\
\hline Flujo de fondos & $-5000,00$ & $-212,25$ & 8378,55 \\
\hline \multicolumn{4}{|c|}{ Los indicadores económicos que arroja los cultivos en El Bolloquito } \\
\hline \multicolumn{2}{|c|}{ Tasa de descuento } & \multicolumn{2}{|c|}{$15 \%$} \\
\hline Valor Neto Actual & S/ 10308,54 & \multicolumn{2}{|c|}{ Estimaciones de la viabilidad } \\
\hline Tasa Interna de Retorno & $27,34 \%$ & VPB & $\mathrm{S} / 6150,82$ \\
\hline Relación Beneficio/Costo & $\mathrm{S} / 1,23$ & VPC & $S / 5000,00$ \\
\hline \multicolumn{4}{|c|}{ Los indicadores económicos que arrojan los cultivos en El Bolloquito son: } \\
\hline Valor Neto Actual (VAN) & & Viable & \\
\hline Tasa Interna de Retorno (TIR) & & Viable & \\
\hline Relación Benficio Costo (BC) & & Viable & \\
\hline
\end{tabular}

del sistema financiero agrario que es del $15 \%$, el ratio $\mathrm{B} / \mathrm{C}$ indica que la inversión es positiva, ya que por cada $S / 1,0$ que se invierte, se recuperan $\mathrm{S} / 1,52$ (Tabla 2).

En cuanto al flujo de caja para los periodos del cultivo del arroz y del cultivo de yuca en Bolloquito, se reporta un flujo de caja negativo de S/ 212,25 para el primero y un flujo de caja positivo de S/ 8378,55 para el segundo. El VAN muestra rentabilidad positiva de S/ 10308,54 , la TIR fue positiva, ya que nos indica que podemos pagar una tasa de interés de hasta $27,34 \%$, superior a la tasa de interés del sistema financiero agrario que es del $15 \%$, el ratio $\mathrm{B} / \mathrm{C}$ fue positivo y nos indica que por cada $S / 1,0$ que se invierte, se recuperan $S / 1,23$ (Tabla 3).

Con respecto al análisis económico-financiera de los componentes arroz y yuca del sistema de producción en las tres localidades en estudio, se observa que en las tres localidades el sistema arroja tasas económicas y financieras favorables al final de la producción de la primera cosecha de arroz y de yuca. En las tres se obtienen un VAN positivo, una TIR superior a la tasa de interés y un coeficiente de beneficio-costo superior a la unidad monetaria de inversión. Las tres localidades presentan viabilidad económica y financiera para el establecimiento de plantaciones de cultivos alimenticios anuales en asociación con pijuayo para fruto y Centrosema de cobertura (Tabla 4).

Los resultados nos permiten inferir que el VAN de El Dorado (S/ 12 559,52), muestra una ligera mayor rentabilidad respecto a los de El Paujil (S/ 12 463,59) y Bolloquito (S/ 10 308,54), la TIR que se puede pagar son similares en El Dorado y El Paujil (40,98 \% y 41,09 \% respectivamente), siendo ambas superiores a la de Bolloquito $(27,34 \%)$. La relación $\mathrm{B} / \mathrm{C}$ también es positiva y similar tanto en El Dorado como en El Paujil (S/ 1,50, S/ 1,52 respectivamente), ambas son ligeramente superiores a la de Bolloquito (S/ 1,23). En general, de acuerdo a los ratios económicos y financieros evaluados en las tres localidades, se demuestra que los componentes yuca y arroz 
Tabla 4. Resumen de las taras económicas y financieras de los componentes arroz (Oryza sativa L.) y yuca (Manihot esculenta Crantz) del sistema de producción en tres localidades de la Carretera lquitos-Nauta, Loreto, Perú.

\begin{tabular}{crrr}
\hline Taras & El Dorado & \multicolumn{1}{c}{ El Paujil } & \multicolumn{1}{c}{ El Bolloquito } \\
\hline VAN & S/ 12559,52 & S/ 12463,59 & $S / 10308,54$ \\
TIR & $40,98 \%$ & $41,09 \%$ & $27,34 \%$ \\
B/C & S/ 1,50 & S/ 1,52 & S/ 1,23 \\
\hline
\end{tabular}

la dinámica de la inundación (List et al., 2019).

\section{CONCLUSIONES}

Las evaluaciones de los componentes arroz y yuca en la localidad El Dorado tiene un VAN S/ 12 559,52, rentabilidad ligeramente superior a las localidades

en el sistema con pijuayo, son viables respecto a los resultados obtenidos. Baluarte et al. (2003) evaluando económica y financieramente sistemas agroforestales secuenciales en suelos de altura, obtuvieron valores para la VAN entre S/ 11901,00 y S/ 29 029,00, para la TIR entre 12,57 \% y $48,50 \%$ y para la relación B/C entre 2,14 y 4,16, concluyendo que los sistemas agroforestales evaluados son factibles y rentables. Por otra parte, Gonzáles \& Acosta (2013), validaron un sistema de producción con cuatro genotipos de pijuayo en suelo de altura, utilizando un sistema secuencial arroz-yuca-yuca-pijuayo, con el que obtuvieron una VAN de S/ 1872,00; una TIR de 15,49 \% y una relación $\mathrm{B} / \mathrm{C}$ de 2,06, considerando viable al sistema de producción. La producción en estos sistemas asegura una viabilidad económica y financiera favorable para el agricultor. Así lo demuestran Ordoñez \& Aloisia da Cruz (2016), en un estudio de viabilidad económica de producción de yuca industrial versus ganado en Sucre, Colombia, reportando para el cultivo de yuca un VAN positivo en comparación con el VAN negativo del ganado.

La TIR encontrada para la yuca fue de 3,41\%, valor superior a la tasa de interés, siendo la TIR encontrada en el ganado inferior a la tasa de interés, indicando que el cultivo de la yuca es económicamente viable. El arroz es un cultivo rentable que se siembra a lo largo de las orillas del río Amazonas (Chibnik, 1994; List et al., 2019), sin embargo, el riesgo de pérdida es alto debido a de El Paujil (S/ 12 463,59) y Bolloquito (S/ 10 308,54); la TIR de El Dorado y El Paujil (40,98 $\%, 41,09 \%$ indican una rentabilidad superior a la de Bolloquito (27,34\%); la relación B/C de El Dorado y de El Paujil (S/ 1,50, S/ 1,52) es ligeramente superior a la de Bolloquito (S/ 1.23).

Los componentes en un sistema productivo de arroz y yuca en la localidad de El Dorado son los más rentables, porque tienen los mejores ratios financieros. Le sigue la localidad de El Paujil y por último, la localidad de Bolloquito.

El cultivo de arroz y yuca en un sistema productivo, en asociación a una plantación de pijuayo, manejados bajo una cobertura de Centrosema, posibilitan ingresos económicos suficientes en los primeros años que viabilizan el éxito en las diferentes localidades.

El estudio de la viabilidad económica y financiera del cultivo de arroz y yuca, en un sistema de producción de pijuayo en el eje de la carrera Iquitos-Nauta, arroja indicadores económicos del VAN, TIR Y B/C viables para todas las localidades.

\section{REFERENCIAS BIBLIOGRÁFICAS}

Álvarez Rodríguez, J.F; Peña Peña, M. 2003. Evaluación financiera y económica para la producción de café bajo el esquema de comercio justo. Tesis de Postgrado. Universidad de los Andes, Facultad de Economía. Bogotá, Colombia. 124pp. 
Baluarte, J.; Acosta, A.; Fernández, A. 2003. Estudio de evaluación económica y financiera de sistemas de producción de copoazú. Instituto Nacional de Innovación Agraria. Estación Experimental Agraria San Roque. Iquitos, Perú. 21pp.

Chibnik, M. 1994. Risky rivers: the economics and politics offloodplain farming in the Amazon. The University of Arizona Press, Tucson. 267pp.

Girón, E.H.2012. Latasainterna de retornoyel valor actual neto como herramientas de evaluación financiera, en proyectos para plantaciones de madera teca. Tesis de Pregrado. Universidad de San Carlos de Guatemala, Facultad de ciencias económicas, Ciudad de Guatemala. 141pp.

Gonzales, R.; Acosta, A. 2013. Validación económica del sistema de producción de pijuayo (Bactris gasipaes H.B.K.) para producción de fruto. Instituto Nacional de Innovación Agraria. Estación Experimental Agraria San Roque. Iquitos, Perú. 12pp.

Holdridge, 1987. Ecología basada en zonas de vida. 5a edición. Instituto de Interamericano de Cooperación para la Agricultura. San José, Costa Rica. 226pp.

INIA. 2014. Labores culturales en el cultivo de yuca, Hoja Divulgativa 17. Instituto Nacional de Innovación Agraria. Estación Experimental Agraria San Roque. Iquitos, Perú. 2pp.

Labarta, R.; White, D.; Leguía, E.; Guzmán, W.; Soto, J. 2007. La Agricultura en la Amazonía Ribereña del Río Ucayali. ¿Una Zona Productiva pero Poco Rentable?. Acta Amazonica. Vol. 37(2): 177-186.

List, G.; Coomes, O. 2019. Repiquetes y riesgo en el cultivo de arroz de Iquitos, Perú. Folia Amazónica, 28(1): 19-32. DOI: https://doi. org/10.24841/fa.v28i1.466

Martínez, A.; Aristizábal, D.; García, F. 2006. Evaluación de un sistema agroforestal en callejones con productores de economía campesina del Piedemonte Araucano y Casanareño. CorpoicaPronatta, Villavicencio, Colombia. 45pp.

Mao, N.; Sánchez, C.; Takayuki, I.; Martínez, D.; Pinzón, M. 2000. Manual de agroforesteria. JICA. San José, Costa Rica. 38pp.

MINAM. 2016. Loreto, cosechando desarrollo en el campo, resultados 2011-2016. Ministerio del Ambiente. Lima, Perú. 7pp.

Musalem, S. 2001. Sistemas agrosilvopastoriles. Universidad Autónoma de Chapingo. División de Ciencias Forestales. Texcoco, México. 120pp. Ordoñez, A.; Aloissia da Cruz, A. 2016. Viabilidad económica de producción de yuca industrial versus ganado en Sucre, Colombia. Revista Ipecege, 2(2): 7-23. DOI: https://doi. org/10.22167/r.ipecege.2016.2.7

Palacios, O. 2010. Arroz INIA "La Esperanza". Programa Nacional de Investigación de Arroz. Estación Nacional de Innovación Agraria "El Porvenir". Tarapoto, Perú. 4pp.

Rivas, T. 2005. Sistemas Agroforestales 1. UACH. 8pp.

SERFOR. 2015. Interpretación de la dinámica de la deforestación en el Perú y lecciones aprendidas para reducirla. Documento de trabajo. Servicio Nacional Forestal y de Fauna Silvestre. Lima, Perú. 40pp.

Smith, J.; Schwartz, J. 2015. La deforestación en el Perú. Cómo las comunidades indígenas, agencias gubernamentales, organizaciones sin fines de lucro y negocios trabajan juntos para detener la tala de los bosques. WWF-PERÚ. 6pp.

Urquiza, J.D.; Burga, R. 2016. Incremento de la deforestación y sus consecuencias en la pérdida de biomasa en los bosques de la región Loreto, 2000-2014. Tesis para optar el grado académico de magister en Ciencias en Gestión Ambiental. Escuela de Posgrado. Universidad Nacional de la Amazonía. Iquitos, Perú 57pp.

Recibido: 16 de febrero de 2020 Aceptado para publicación: 22 de mayo de 2020

Esta obra está bajo una Licencia Creative Commons Atribución-NoComercial-SinDerivar 4.0 Internacional. 